\title{
On possible approaches to visualizing the process of selective laser melting
}

\author{
A.A. Molotkov ${ }^{1, A, B}$, O.N. Tretiyakova ${ }^{2, A, B}$ \\ A Moscow aviation Institute (national research University) MAI \\ B LLC "PROMIS LAB” of the group of companies "Lasers and Equipment" \\ 1 ORCID: 0000-0002-9335-5219, karacerr@gmail.com \\ 2 ORCID: 0000-0003-0256-4558, tretiyakova olga@mail.ru
}

\begin{abstract}
This paper deals with the visualization of the previously simulated by the authors selective laser melting process in order to simplify the analysis of the results and the selection of technological parameters of the additive production unit. The article presents two possible approaches for visualization of the selective laser melting process and supported functions, which simplify the work and research in the framework of the new technology. In the twodimensional visualization mode, the emphasis is on the possibility of a more detailed study of the process. In a three-dimensional there is the ability of the broader scope and to see the big picture.
\end{abstract}

Keywords: 3D model, visualization, mathematical model, selective laser.

\section{Introduction}

Today, the development of powder metallurgy and laser optics allows not only to conduct high-precision research [1], but also to develop new production methods. The technology of layer-by-layer selective laser melting SLM (selective laser melting) is a new technology of additive manufacturing, which allows to obtain products of complex shapes, which can not be created with the help of classical production methods. It is most in demand in the aerospace industry [2], for example, when creating parts from various types of steels, aluminum and titanium alloys [3], and the biomedical industry [4]. It is worth noting that this technology is implemented on Russian equipment, for example, in the ML6-1 unit produced by the group of companies "Lasers and equipment", fig. 1.

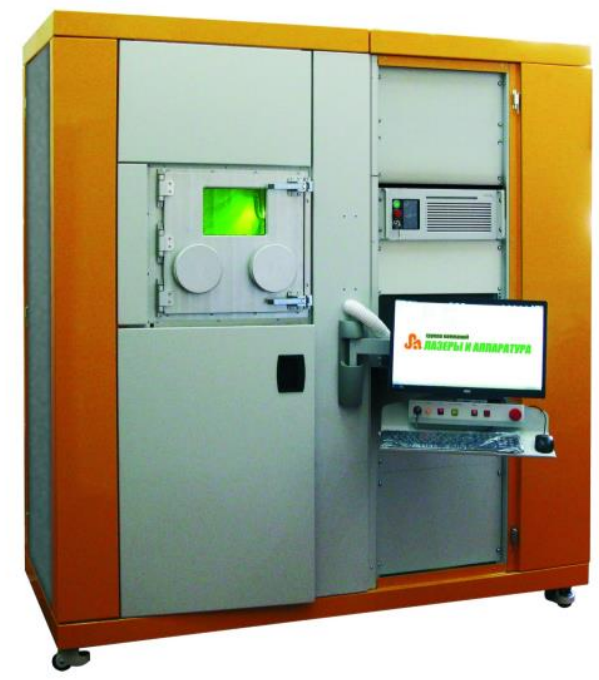

Fig. 1. Experimental equipment ML-6-1. 


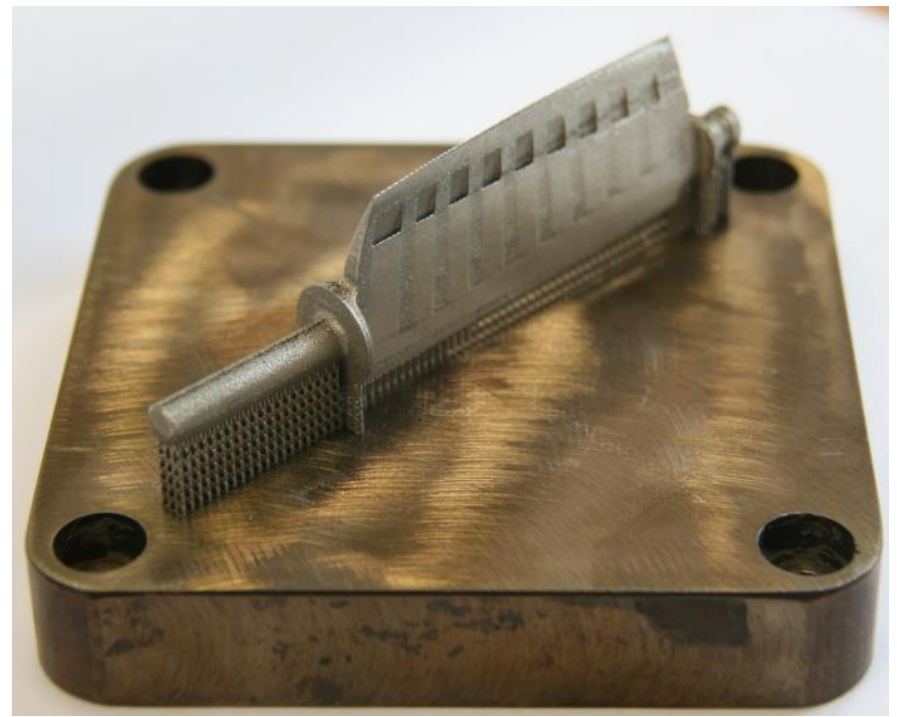

Fig. 2. Detail made on equipment ML-6-1.

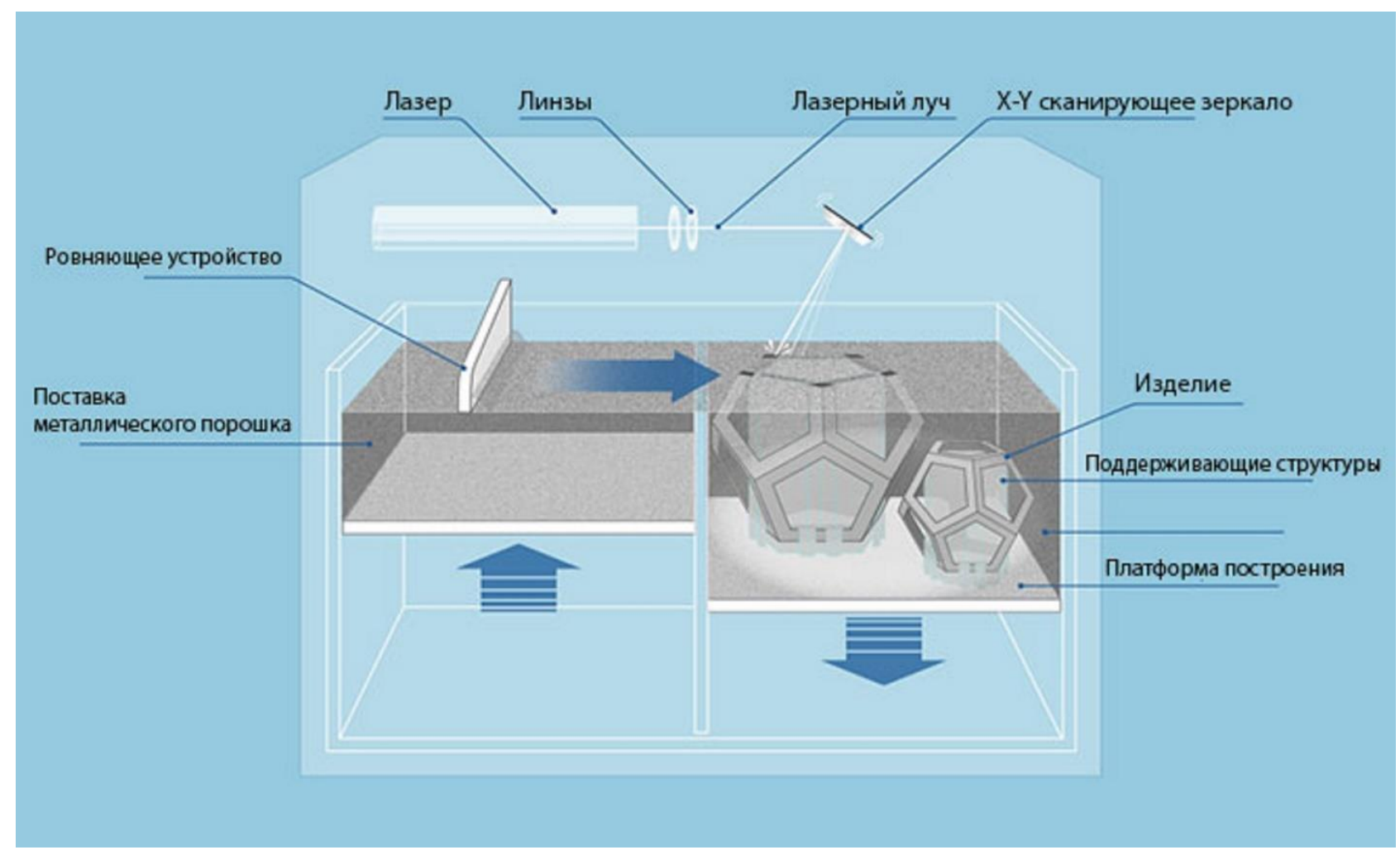

Fig. 3. Schematic diagram of SLM process. [9]

The narrowness of the technological parameters is caused by the complexity of the geometry of manufactured parts, fig. 2, and the complexity of the manufacturing process itself, fig. 3 . These aspects lead to the need for numerical simulation [5-7]. In the first approximation, the problem of modeling the SLM process is reduced to solving the boundary value problem of thermal conductivity taking into account the phase transitions of the first kind (melting and solidification).

The paper [8] describes in more detail the numerical simulation of heat transfer in the process of SLM and comparison with the calculations of other authors. On the basis of the obtained results, a software package was created, including:

1. calculation of heat transfer in SLM process, 2. visualization of temperature field simulation results. An important part of the software package are the visual components responsible for displaying the results. The program provides two modes of visualization: two-dimensional and three-dimensional. 


\section{Mathematical model}

In the first approximation, a mathematical model of the process of heating a metal object with a mobile heat source is created for modeling the SLM process. The heat source is a unimodal Gaussian beam of laser radiation. Some of the incident radiation is reflected. The heated material undergoes melting and hardening processes during the calculations.

To set the Gaussian beam, the surface of the heated body was divided into several regions. At each region, a boundary condition of the second kind was set, where the incoming heat flow was calculated through the intensity of the radiation incident on this region at a certain point in time. Thus, the Gaussian function of the transverse spatial distribution of the laser beam power density is approximated by a piecewise linear function. From the ratios (1-2)

$$
\begin{gathered}
I=I_{0} e^{-\frac{2 r^{2}}{\omega^{2}}} \\
P_{0}=2 \pi \int_{0}^{\infty} I(r) r d r=\frac{\pi}{2} \omega^{2} I_{0},
\end{gathered}
$$

have

$$
I(r)=\frac{2 P_{0}}{\pi \omega^{2}} e^{-\frac{2 r^{2}}{\omega^{2}}},
$$

where $\omega$ - the characteristic radius of the beam (the distance from the center of the beam at which the intensity decreases $e^{2}$ times), $I_{O}$ - maximum intensity, $P_{O}$ - laser power, $I$ - intensity, $r$ - distance to the center of the spot. Thus we come to the dependence of the intensity at a particular point in space.

For the problem to be solved it was necessary to consider the significant influence of the reflection of the radiation from the metal surface. The formula for radiation intensity, which is used by us in further calculations, is as follows

$$
I(r)=(1-R) \frac{2 P_{0}}{\pi \omega^{2}} e^{-\frac{2 r^{2}}{\omega^{2}}}
$$

where $R$ - the reflection coefficient of the metal surface.

The basis of the mathematical model, as already mentioned, is the equation of thermal conductivity, which describes the distribution of temperature in a given region of space and its change in time.

$$
p C(T) \frac{\partial T}{\partial t}=K\left(\frac{\partial^{2} T}{\partial x^{2}}+\frac{\partial^{2} T}{\partial y^{2}}+\frac{\partial^{2} T}{\partial z^{2}}\right)+q
$$

where $p$ - density, $C(T)$ - a function of the dependence of heat capacity on temperature, $K$ the coefficient of thermal conductivity, $T$ - temperature, $x, y, z$ - spatial variables, $t$ - time, $q$ the bulk density of heat sources at time $t$ at point $x$.

With initial conditions

and mixed boundary conditions

$$
T(x, y, z, 0)=300^{0} \mathrm{C}
$$

$$
\left\{\begin{array}{l}
T(0, y, z, t)=300^{0} C \\
T\left(l_{x}, y, z, t\right)=300^{\circ} C \\
T(x, 0, z, t)=300^{\circ} C \\
T\left(x, l_{y}, z, t\right)=300^{\circ} C \\
q(x, y, 0, t)=Q(x, y, z, t) \\
T\left(x, y, l_{z}, t\right)=300^{\circ} C
\end{array}\right.
$$


The heat flux at the boundary is calculated by using the formula (4).

This differential boundary value problem using numerical differentiation formulas:

$$
\begin{gathered}
\frac{\partial T}{\partial t}=\frac{T_{i, j, m}^{n+1}-T_{i, j, m}^{n}}{\Delta t} \\
\frac{\partial^{2} T}{\partial x^{2}}=\frac{K}{h_{x}^{2}}\left(T_{i+1, j, m}^{n}-2 T_{i, j, m}^{n}+T_{i-1, j, m}^{n}\right) \\
\frac{\partial^{2} T}{\partial y^{2}}=\frac{K}{h_{y}^{2}}\left(T_{i, j+1, m}^{n}-2 T_{i, j, m}^{n}+T_{i, j-1, m}^{n}\right) \\
\frac{\partial^{2} T}{\partial z^{2}}=\frac{K}{h_{z}^{2}}\left(T_{i, j, m}^{n}-2 T_{i, j, m}^{n}+T_{i-1, j, m}^{n}\right)
\end{gathered}
$$

it is transformed into a finite difference boundary value problem of the form

$$
\begin{aligned}
& p C \frac{T_{i, j, m}^{n+1}-T_{i, j, m}^{n}}{\Delta t}=\frac{K}{h_{x}^{2}}\left(T_{i+1, j, m}^{n}-2 T_{i, j, m}^{n}+T_{i-1, j, m}^{n}\right)+ \\
& +\frac{K}{h_{y}^{2}}\left(T_{i, j+1, m}^{n}-2 T_{i, j, m}^{n}+T_{i, j-1, m}^{n}\right)+\frac{K}{h_{z}^{2}}\left(T_{i, j, m+1}^{n}-2 T_{i, j, m}^{n}+T_{i, j, m-1}^{n}\right)+Q
\end{aligned}
$$

The model of phase transitions is changed in comparison with the work [10] by means of replacement:

Thus, have

$$
\frac{H_{i, j, m}^{n+1}-H_{i, j, m}^{n}}{\Delta t}=p C \frac{T_{i, j, m}^{n+1}-T_{i, j, m}^{n}}{\Delta t}
$$

$$
\begin{aligned}
& \frac{H_{i, j, m}^{n+1}-H_{i, j, m}^{n}}{\Delta t}=\frac{K}{h_{x}^{2}}\left(T_{i+1, j, m}^{n}-2 T_{i, j, m}^{n}+T_{i-1, j, m}^{n}\right)+ \\
& +\frac{K}{h_{y}^{2}}\left(T_{i, j+1, m}^{n}-2 T_{i, j, m}^{n}+T_{i, j-1, m}^{n}\right)+\frac{K}{h_{z}^{2}}\left(T_{i, j, m+1}^{n}-2 T_{i, j, m}^{n}+T_{i, j, m-1}^{n}\right)+q
\end{aligned}
$$

where $H$ - the enthalpy, $K$ - the coefficient of thermal conductivity, $h$ - the spatial step, $T$ - the temperature, $\Delta t$ - the time step, $i, j, m$ - the spatial coordinate indices, $n$ - the time index. And the temperature dependence on enthalpy $(H)$ has the form [10].

$$
T=\left\{\begin{array}{cc}
H / C_{S}, & H \leq C_{s} T_{m} \\
T_{m}, & C_{s} T_{m}<H<C_{s} T_{m}+H_{m}, \\
T_{m}+\frac{H-C_{s} T_{m-H} H_{m}}{C_{l}}, & C_{s} T_{m}+H_{m} \leq H
\end{array}\right.
$$

where $T_{m}$ - melting point, $C_{s}$ - heat capacity before the phase transition, $C_{l}$ - heat capacity after the phase transition.

This explicit finite difference scheme (6) has the first order of accuracy in time and the second in space.

The heat capacity of the material varies with temperature, this dependence is taken into account by changing the model of temperature recalculation through enthalpy. The method of 
temperature recalculation by means of enthalpy is somewhat modified by us, in comparison with the work [10].

If we have data on the dependence of the bulk heat capacity of the material on the temperature $C_{1}-T_{1}, C_{2}-T_{2}, \ldots, C_{n}-T_{n}$, then, determining recursively the enthalpy value corresponding to the specified temperatures

$$
\begin{gathered}
H_{1}=C_{1} * T_{1} \\
H_{2}=H_{1}+C_{2} * T_{2} \\
\cdots \\
H_{n-1}=H_{n-2}+C_{n-1} * T_{n-1} \\
H_{n}=H_{n-1}+C_{n} * T_{n}
\end{gathered}
$$

and, having previously calculated these values, we come to a new model of temperature recalculation from enthalpy

$$
T=\left\{\begin{array}{cc}
H / C_{1}, & H \leq H_{1} \\
T_{1}+\frac{H-H_{1}}{\left(C_{2}-C_{1}\right) / 2}, & H_{1}<H<H_{2} \\
T_{2}+\frac{H-H_{2}}{\left(C_{2}-C_{1}\right) / 2}, \ldots \ldots \ldots \ldots \ldots \ldots \ldots \ldots \\
\cdots \ldots \ldots \ldots \ldots \ldots \ldots+H_{n-1} \\
T_{n-2}+\frac{H-H_{n-2}}{\left(C_{n-1}-C_{n-2}\right) / 2}, & H_{n-2}<H<H<H_{n-1} \\
T_{n-1}+\frac{H-H_{n-1}}{\left(C_{n}-C_{n-1}\right) / 2}, & H_{n-1}<H<H_{n} \\
T_{m}+\frac{H-C_{s} T_{m-} H_{m}}{C_{l}}, & H_{n}<H<H_{n}+H_{m} \\
&
\end{array}\right.
$$

\section{Software implementation}

To simulate heat transfer in the SLM process, SLMT1 programs were created to calculate the temperature distribution in the melt bath region and SLMT2 to find the temperature field in the entire part.

The SLMT1 program served as the basis for the thermal simulation module (hereinafter SimulationLib) for the LaserAprBuildProcessor software complex-a part of the software of the laser $3 \mathrm{D}$ printing machine using the selective laser fusion ML6-1 technology Produced by the Lasers and equipment group of companies.

LaserAprBuildProcessor is a software complex, those tasks are: 1) providing a comprehensive assistance to the process engineer and the operator of the machine, 2) the division of a part model into layers, which are the input data for the operation of the machine, 3) filling the layers with a plurality of segments-laser trajectories, 4) building supports, 5) generation of laser modes

SimulationLib (SLMT2) is a thermal simulation module, a companion program that makes it easier for a process engineer to perform his or her duties.

Unlike SLMT1, SimulationLib calculates the temperature distribution in the entire part rather than in the melt bath area. In the new program SLMT2 became possible to consider the trajectory of the laser beam, the geometry of the part, sprinkling layers were taken into account the dependence of thermophysical parameters of material temperature.

Ray paths are segments defined by two points (the start point and the end point of the segment). SimulationLib gets them, along with the geometry of the part from the external environment of LaserAprBuildProcessor. 


\section{General information about the program code}

The program code is written in C\# using .NET platform technologies (WPF). The installation program was created using NSIS (Nullsoft Scriptable Install System). The program is designed to run on Microsoft Windows and requires the .NET Framework 4.7. For visualization, the capabilities of the open library SharpGL for C\# are actively used, which allows a user to easily use the capabilities of OpenGL. Application of MVVM (Model-View-ViewModel) architectural design pattern allowed to separate application logic from the visual part (representation) and to achieve a clearer and more meaningful structure. The input data are CLI (Common Layer Interface) files, a universal format for entering geometric data into model fabrication systems based on layer fabrication technologies (LMT). It is suitable for systems using layer-by-layer photo-curing of resin, sintering or binding of powder, cutting of sheet material, solidification of molten material, and any other systems that create models in layers. This format is actively used in the additive manufacturing industry. Data of this format is obtained as a result of the work of various programs for the preparation and editing of data in the STL format (from the English. stereolithography) for additive manufacturing. A striking example of such a program is Materialize Magics.

\section{Two-dimensional visualization}

In 2-D mode, the part is displayed in layers, the projections of the supports necessary to compensate for the thermal stresses formed during the process and prevent deformation. If necessary, the program:

1. Creates one or more equidistants outlining the contour of the part, which allows a user to bypass the part along the contour of the laser in different modes. This allows a user to achieve a higher surface quality later.

2. Splits the interior space into areas of varying area and shading them. Each resulting bar-line is the trajectory of the laser. The procedure of such "shading" is a stage of preparation of the technical specification of the machine.

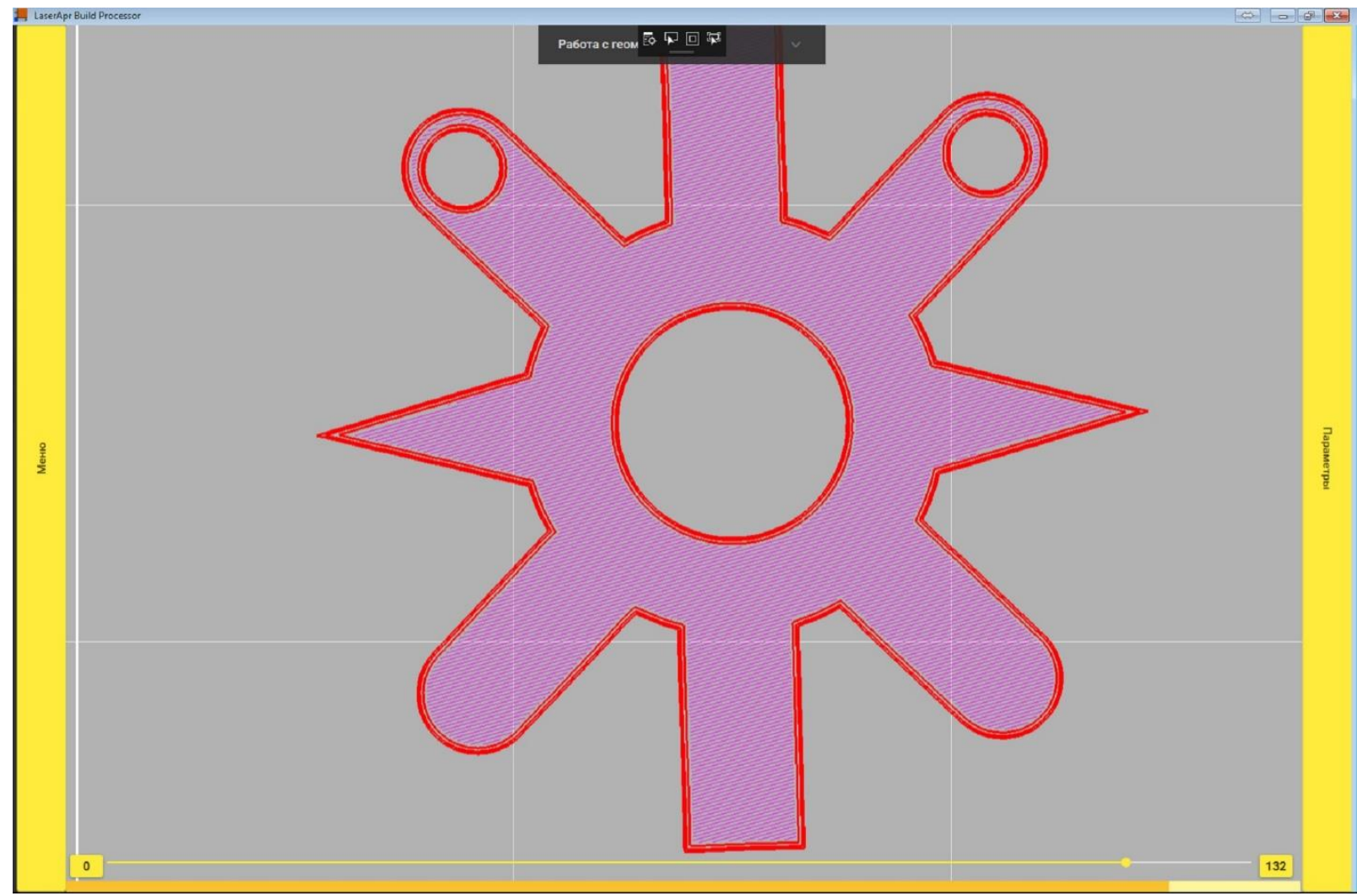

Fig. 4. The circuit details. 
In consequence, on each layer, fig. 4, after the simulation, the temperature field is superimposed, fig. 5, fig. 6. The calculation takes into account the trajectory of the laser, its power, speed, form of radiation distribution, thermophysical parameters of the material, powder deposition. Information about each step of the numerical calculation is stored in a buffer, which allows a user to determine the temperature value at any time of the upcoming fusion process for each layer. To simplify the analysis of the results, the function of creating custom palettes is provided, with which the technologist can determine the number of color intervals, their length, color, as well as which fill option (gradient or solid) for each interval to use. It is possible to dynamically change the monitored interval using a two-finger slider, which allows a user to focus on a specific area of interest to the technologist. Since even in layers with a high grouping of objects and their dense shading, the number of displayed elements is relatively low, performance problems do not arise even on weak systems. On the contrary, this problem is acute in three-dimensional mapping.

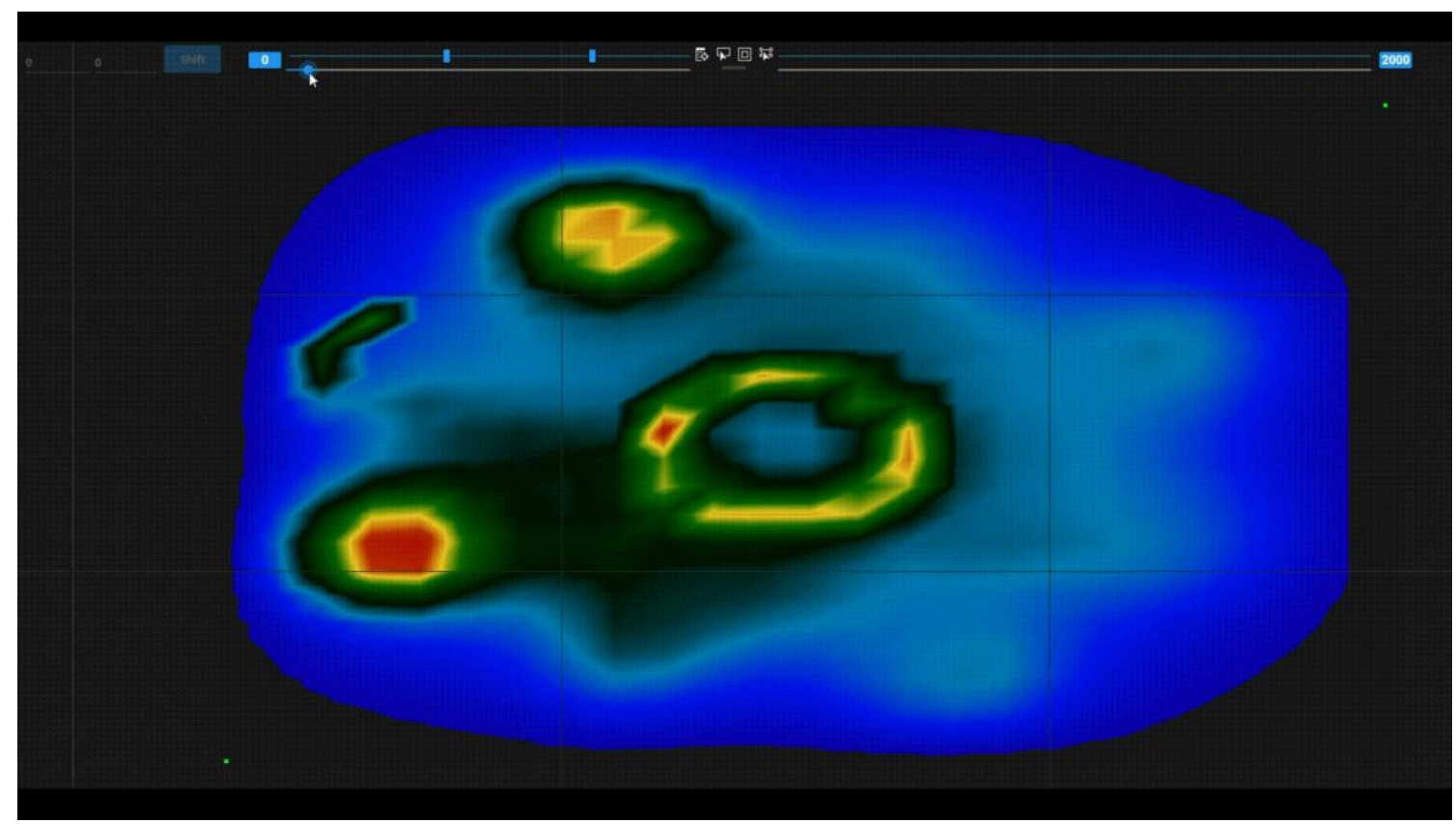

Fig. 5. Visualization of the heating of the part in the manufacturing.

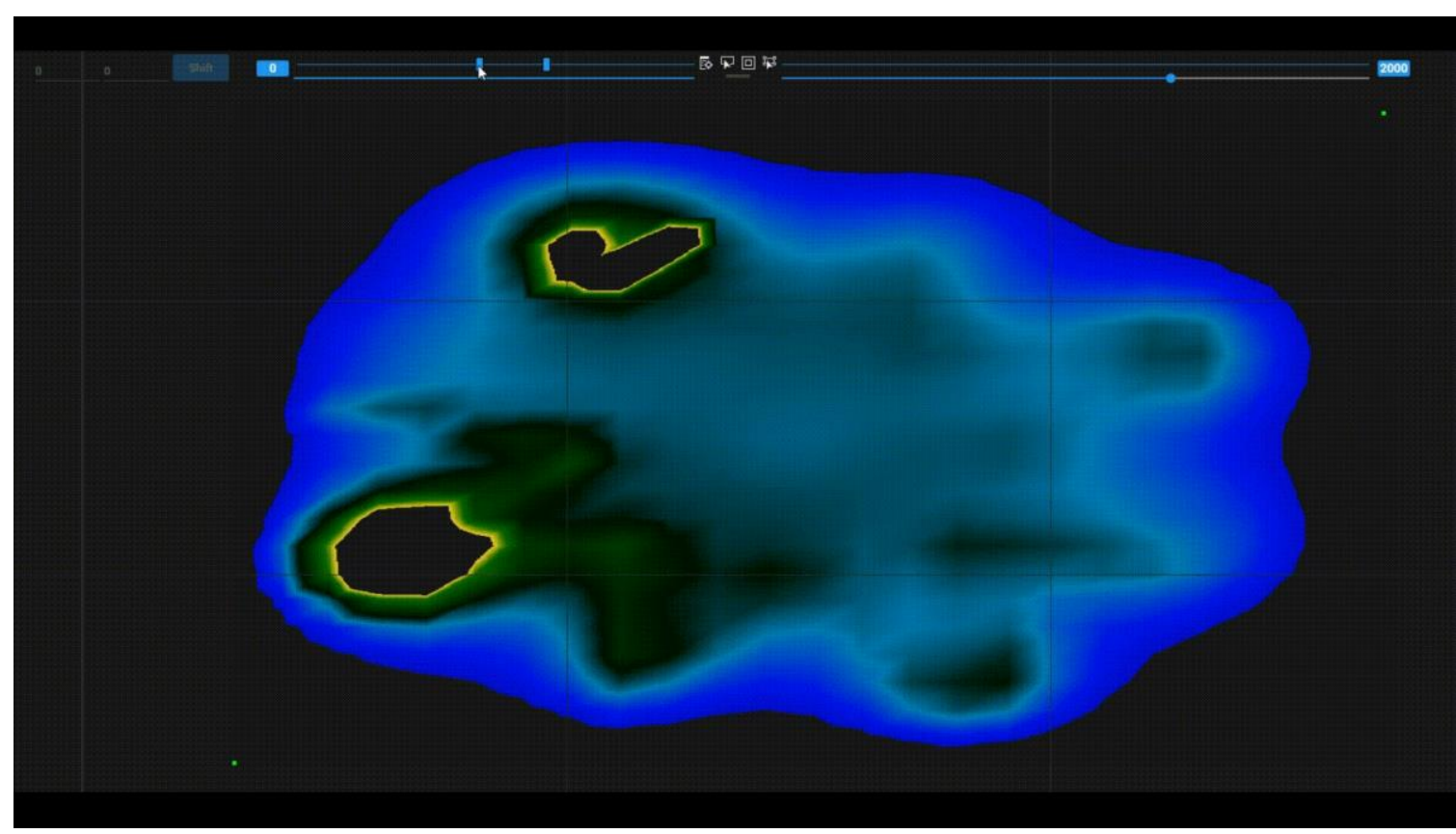

Fig. 6. The selection of the critical zone of the greatest heating is shown in red. 


\section{Three-dimensional visualization}

In 3-D mode, all layers are displayed together, allowing a user to see the part in its original form, Fig. 7. It is possible to view temperatures, Fig. 8, Fig. 9, but unlike the 2-D mode, the temperature field can be viewed in three projections. The colors displayed in this mode are superimposed on three perpendicular secant planes. When each of these planes moves, parts that interfere with the view are removed from the display. Thus, in 3-D mode, the operator is able to see the temperatures not only on the XoY plane, but also in the YoZ and XoZ planes. Since the number of displayed graphical primitives based on the layer map exceeds half a million for large parts in some cases, the displayed geometry is simplified before rendering, so that the difference is barely noticeable to the operator. Simplification is implemented according to three principles:

1. Cutting a three-dimensional model of the manufactured part occurs in the data preparation programs, as indicated earlier. Because the geometry of a layer is determined by looking for the intersection of the triangular primitives that make up the object model, in the resulting file, very often even straight lines represent more than one object. Such segments, without the risk of losing accuracy, can be re-combined into one, thereby reducing the number of primitives displayed in the aftermath. The computational complexity of this simplification is $O(N)$, where $N$ is the number of elements in the layer, and is applied only once.

2. Because of the high demands placed on the parts produced in the industry, the lines of varying degrees of curvature are approximated by straight lines of small dimensions, which allow maintaining high surface quality. When visualizing, this accuracy is often unnecessary. Therefore, it is not the original layer map that is used for the display, but its simplified version. If for two segments the beginning of one segment coincides with the end of the second, and one segment lies at a slight angle to the other (if this angle is less than a predetermined value, depending on the distance between the point of view and the segments in question), then such segments can be replaced by one in the simplified version of the layer map. Since the segments in the layer are in order and only the adjacent segments need to be compared, the computational complexity is also $O(N)$, where $N$ is the number of elements in the layer.

3. Most often, any two adjacent layers do not differ much. This allows, with a small approximation, to exclude entire layers from the simplified map. Layers are considered dissimilar if there are segments in one layer that are at a great distance from all segments of another layer. The computational complexity of comparing layers to determine "similarity" is $O(N * M)$, where $N$ and $M$ are the number of elements in the two layers being compared. It is worth noting that a special numerical mark equal to the approximation value is put in accordance with each layer, after which the differences in the layers are invisible and this layer potentially should not be drawn - this allows to further reduce the number of side calculations.

The second and third simplification methods are applied when a user change the display scale. Thus, on zoom out, the number of displayed primitives in most cases decreases, which reduces the load on the system and speeds up the program. On zoom out, the detail of the displayed part increases, which allows a user to avoid losses in information content. The result is to increase the frame rate for large and complex parts from an average of 7 to 15 FPS (frames per second). Despite the fact that 15 FPS is quite a small number, this is quite enough, due to the fact that purely stationary objects are displayed and the movement is represented only in the form of rotation and movement of the camera, movement of secant planes, changes in the number of displayed layers. 


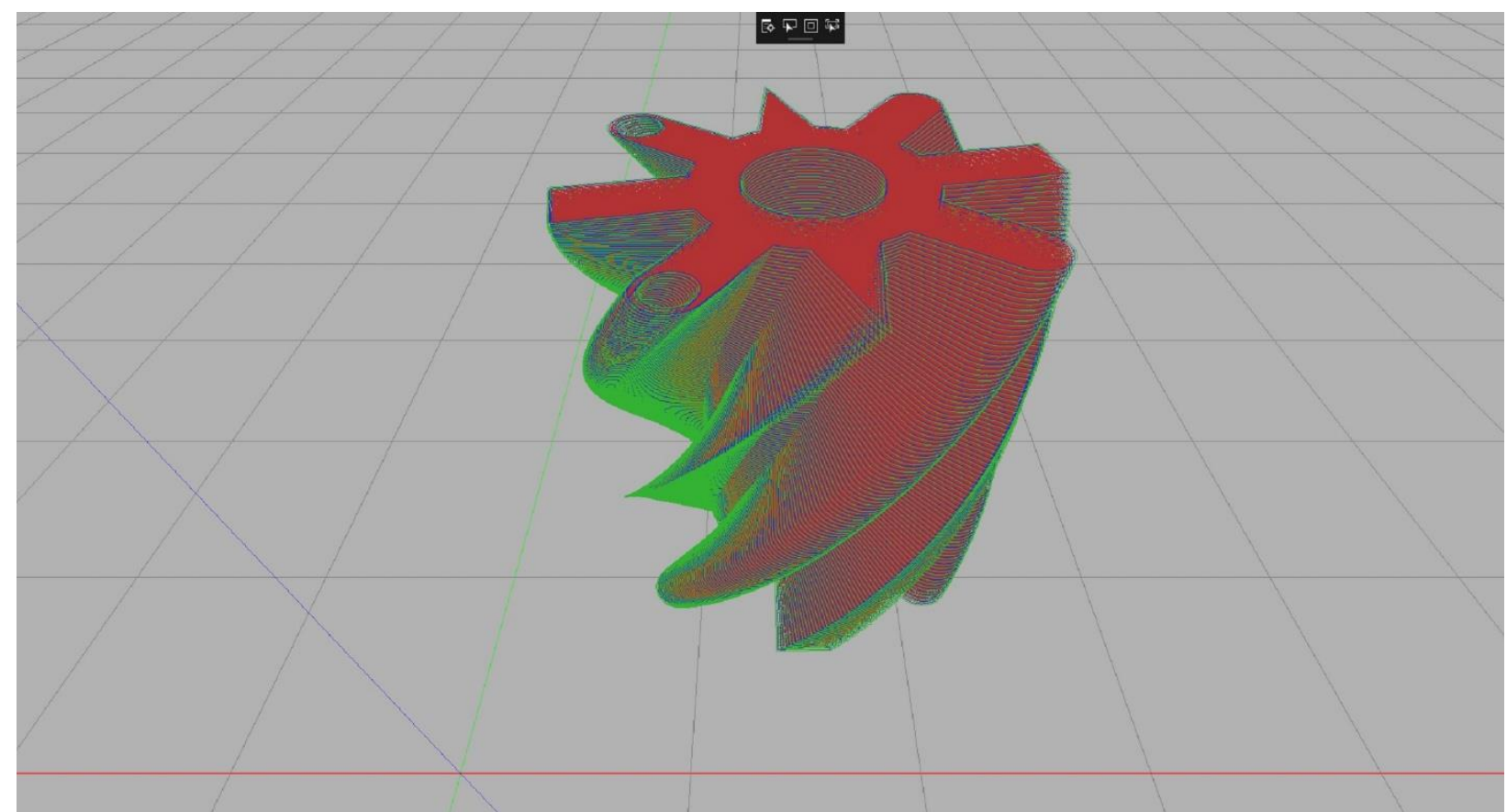

Fig. 7. Three-dimensional model of the created part.

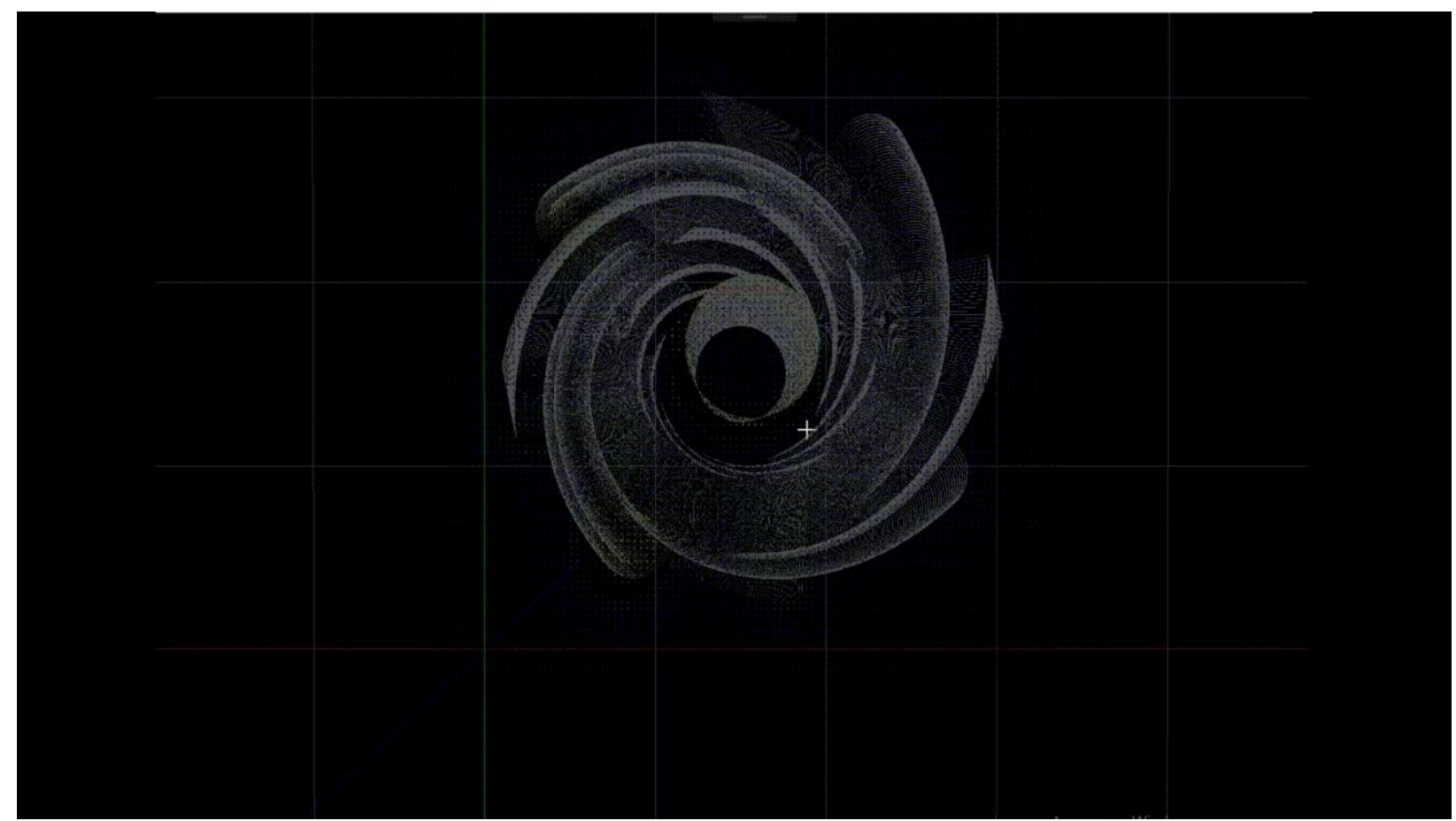

Fig. 8. Part model with superimposed point-to-point temperature display model. 


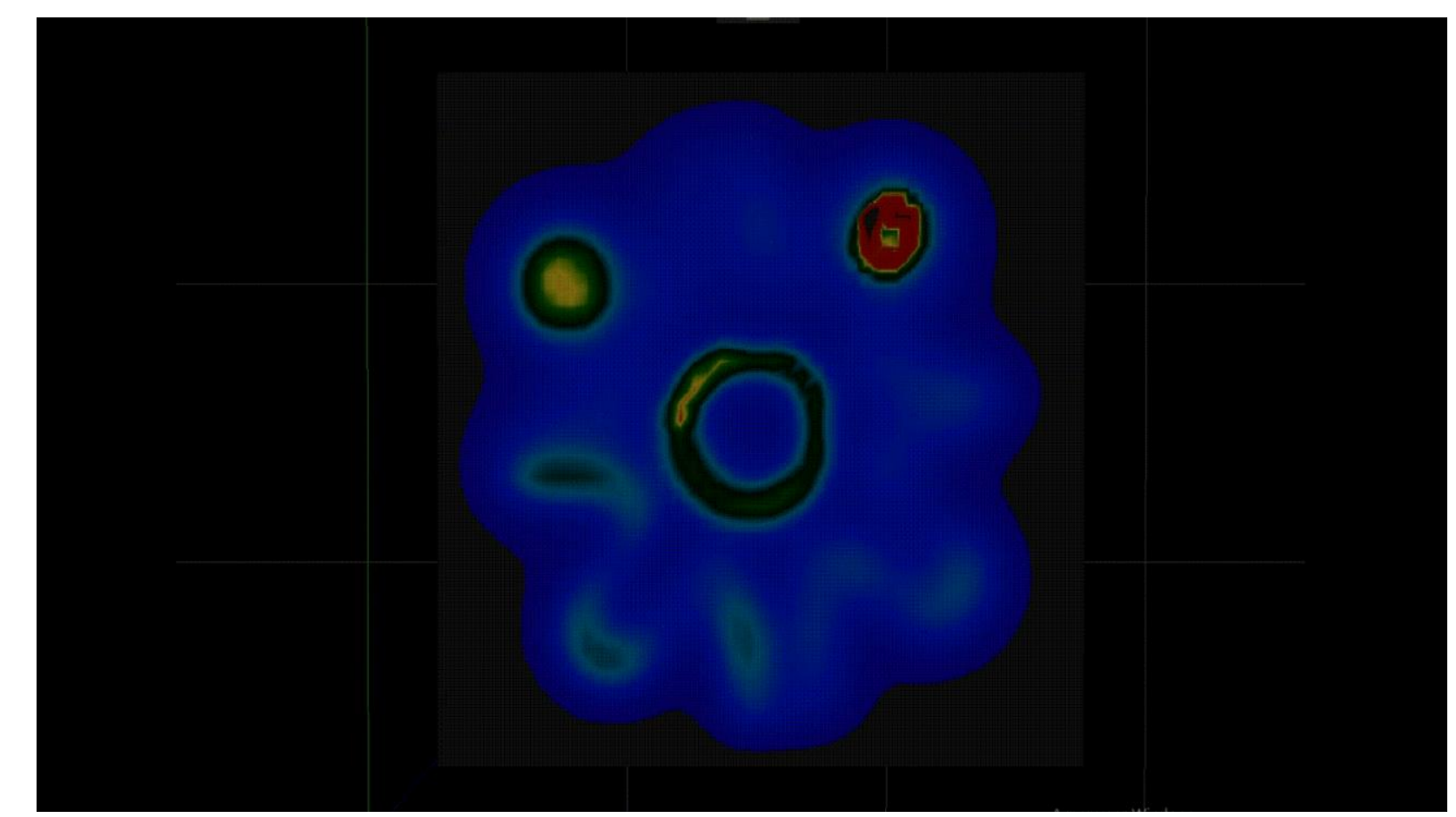

Fig. 9. A model of a part with a cutting plane on which the calculated temperature field is superimposed. Intermediate temperature values are obtained by linear interpolation.

\section{Advantages and disadvantages}

Most often, thermal calculations and similar numerical studies are carried out in special programs, such as products of the Ansys and Comsol family [11]. Creating custom user models of the SLM process in these systems is very difficult, setting all the geometry in manual mode requires an incredible amount of time, and automation of this process requires a high knowledge of specific languages, such as the parametric APDL language specially developed for use in Ansys, and a high level of skills programming. Therefore, such systems have their own solutions to this problem; we wrote in more detail about the differences between these models from ours in [8].

Here it is worth to dwell on the differences in visualization. The Ansys solution for SLM process the received temperature is displayed on the surface of the created items in the appropriate color, to reach values in depth details are not so simple, this can be achieved by pre-split items apart in the graphical editor or by using APDL, here we come again to the necessity knowledge of special of the language. There are problems with setting the color palette, by default the colors in the palette are set, and the interval to which the colors correspond is tied to the maximum and minimum temperature. For all time of use of this product, it is convenient to adjust a palette at us and it did not turn out. In our work, the user can easily pick up a color palette, bind colors to certain temperatures or temperature intervals, highlight areas of interest, use piecewise linear or gradient view of painting, using secant temperature sensors at any point at any time. All these features are available to the technologist through the user interface, which significantly reduces the requirements for the level of training of the user. Also, our program is included in the software for installation of Russian production ML-6-1. The process of calculation and display of its results works asynchronously, which allows at this time to continue to use other functions of the software of the machine or to conduct research on a third-party personal computer (PC).

Among the main disadvantages of the presented implementation of the solution at this stage of work, is that the calculation is mainly single-threaded, which does not allow full use of the computational capabilities of multi-core processors. In more professional programs Ansys and Comsol much attention is paid to the appearance of the work and visualization of the cal- 
culation, texturing, working with lighting, our solution visually looks more rough. Improving the texture quality is possible, but it makes the program code more cumbersome, and the work in General more time-consuming, which at this stage of the work we consider impractical.

\section{Conclusion}

As a result, the creation of two display modes previously developed model was able to simplify the process of research and analysis of the results. Extensive, but not yet all-encompassing functionality allows technologists to see the fullness of the information obtained during the simulation of the SLM process, to see the dynamics of what is happening, to track the areas of interest. The additional ability to use these two modes simultaneously (with the output of the picture on one or more screens) allows a user to simultaneously see the picture as a whole and concentrate on individual, local areas.

\section{Acknowledgments}

The authors Express their gratitude to the management of the group of companies "Lasers and Apparatus" for assistance in the material and technical support of experimental studies and modeling of the process under consideration.

\section{References}

[1] A.V. Vedyashkina, B.S. Rinkevichyus, I.N. Pavlov, I.L. Raskovskaya, A.V. Tolkachev. Optical-electronic complex for investigation of the processes of heat and mass transfer by laser contactless method. Scientific Visualization, 2019, volume 11, number 3, pages 43 - 53, DOI: $10.26583 /$ sv.11.3.04.

[2] P. Rometsch, Q. Jia, K. Yang, X. Wu. Aluminum alloys for selective laser melting - towards improved performance. Additive Manufacturing for the Aerospace Industry, 2019, Pages 301-325

[3] M. Mazur, M. Leary, M. McMillan, S. Sun, D.Shidid, M. Brandt. Mechanical properties of $\mathrm{Ti}_{6 \mathrm{Al}} \mathrm{V}$ and AlSi12Mg lattice structures manufactured by Selective Laser Melting (SLM) Laser Additive Manufacturing. Materials, Design, Technologies, and Applications. Woodhead Publishing Series in Electronic and Optical Materials, 2017, Pages 119-161

[4] K. Vanmeensel, K. Lietaert, B. Vrancken, S. Dadbakhsh, X. Li, J. Kruth, P. Krakhmalev, I. Yadroitsev, J. Humbeeck. Additively manufactured metals for medical applications. Additive Manufacturing, Materials, Processes, Quantifications and Applications, 2018, Pages 261-309

[5] C. Bruna-Rosso, A. Demir, B. Previtali. Selective laser melting finite element modeling: Validation with high-speed imaging and lack of fusion defects prediction. Materials \& Design. Volume 156, 15 October 2018, Pages 143-153

[6] H. Ali, H. Ghadbeigi, K. Mumtaz. Residual stress development in selective laser-melted Ti6Al4V: a parametric thermal modelling approach. The International Journal of Advanced Manufacturing Technology. July 2018, Volume 97, Issue 5-8, pp 2621-2633

[7] E. Mirkoohi, D. Seivers , H. Garmestani , S. Liang. Heat Source Modeling in Selective Laser Melting. Materials 2019, 12, 2052.

[8] Lebyodkin I. F., Molotkov A. A., Tretiyakova O. N. Matematicheskoe modelirovanie slozhnogo teploobmena pri razrabotke lazernyh SLM tekhnologij // Trudy MAI: Elektronnyj zhurnal, ISSN:1727-6924, №101, 2018.[In Russian]

[9] SLM (Selective Laser Melting)-3D printing technology [Electronic resource] // 3d.globatek.ru: [website]. URL: http://3d.globatek.ru/3d printing technologies/slmtech/Gusarov 
[10] A.V., Yadroitsev I., Bertrand Ph., Smurov I. Model of Radiation and Heat Transfer in Lazer-Powder Interaction Zone at Selective Lazer Melting // Journal of Heat Transfer, 2009, vol. 131: 072101-1-9.

[11]K.-H. Leitz, P. Singer, A. Plankensteiner, B. Tabernig, H. Kestler, L. S. Sigl. ThermoFluiddynamical Modelling of Laser Beam-Matter Interaction in Selective Laser Melting. Excerpt from the Proceedings of the 2016 COMSOL Conference in Munich. URL: https://www.comsol.co.in/paper/download/359081/leitz paper.pdf 